\title{
Kinetic Analysis of Hexose Conversion to Methyl Lactate by Sn Beta: Effects of Substrate Masking and of Water
}

Tosi, Irene; Riisager, Anders; Taarning, Esben; Jensen, Pernille Rose; Meier, Sebastian

Published in:

Catalysis Science \& Technology

Link to article, DOI:

10.1039/C8CY00335A

Publication date:

2018

Document Version

Peer reviewed version

Link back to DTU Orbit

Citation (APA):

Tosi, I., Riisager, A., Taarning, E., Jensen, P. R., \& Meier, S. (2018). Kinetic Analysis of Hexose Conversion to Methyl Lactate by Sn Beta: Effects of Substrate Masking and of Water. Catalysis Science \& Technology, 18(8), 2137-2145. https://doi.org/10.1039/C8CY00335A

\section{General rights}

Copyright and moral rights for the publications made accessible in the public portal are retained by the authors and/or other copyright owners and it is a condition of accessing publications that users recognise and abide by the legal requirements associated with these rights.

- Users may download and print one copy of any publication from the public portal for the purpose of private study or research.

- You may not further distribute the material or use it for any profit-making activity or commercial gain

- You may freely distribute the URL identifying the publication in the public portal 


\section{Accepted Manuscript}

This article can be cited before page numbers have been issued, to do this please use: I. Tosi, A. Riisager,

E. Taarning, P. Jensen and S. Meier, Catal. Sci. Technol., 2018, DOI: 10.1039/C8CY00335A.

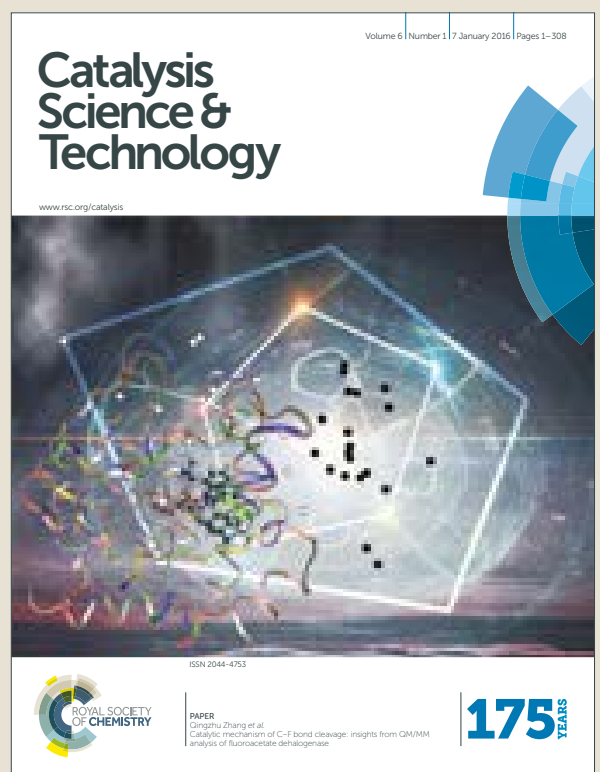

This is an Accepted Manuscript, which has been through the Royal Society of Chemistry peer review process and has been accepted for publication.

Accepted Manuscripts are published online shortly after acceptance, before technical editing, formatting and proof reading. Using this free service, authors can make their results available to the community, in citable form, before we publish the edited article. We will replace this Accepted Manuscript with the edited and formatted Advance Article as soon as it is available.

You can find more information about Accepted Manuscripts in the author guidelines.

Please note that technical editing may introduce minor changes to the text and/or graphics, which may alter content. The journal's standard Terms \& Conditions and the ethical guidelines, outlined in our author and reviewer resource centre, still apply. In no event shall the Royal Society of Chemistry be held responsible for any errors or omissions in this Accepted Manuscript or any consequences arising from the use of any information it contains. 


\title{
Catalysis Science \& Technology
}

3Received 00th January 20xx Accepted 00th January 20xx

DOI: $10.1039 / x 0 x \times 00000 x$

www.rsc.org/

\section{Kinetic Analysis of Hexose Conversion to Methyl Lactate by Sn Beta: Effects of Substrate Masking and of Water ${ }^{\dagger}$}

\author{
Irene Tosi, ${ }^{a}$ Anders Riisager, ${ }^{a *}$ Esben Taarning, ${ }^{b}$ Pernille Rose Jensen ${ }^{c}$ and Sebastian Meier ${ }^{a *}$
}

\begin{abstract}
Simple sugars bear promise as substrates for the formation of fuels and chemicals using heterogeneous catalysts in alcoholic solvents. Sn-Beta is a particularly well suited catalyst for the cleavage, isomerization and dehydration of sugars into more valuable chemicals. In order to understand these processes and save resources and time by optimising them, kinetic and mechanistic analyses are helpful. Herein, we study substrate entry into the Sn-Beta catalysed methyl lactate process using abundant hexose substrates. NMR spectroscopy is applied to show that the formation of methyl lactate occurs in two kinetic regimes for fructose, glucose and sucrose. The majority of methyl lactate is not formed from the substrate directly, but from methyl fructosides in a slow regime. At $160{ }^{\circ} \mathrm{C}$, more than $40 \%$ of substrate carbon are masked (i.e. reversibly protected in situ) as methyl fructosides within few minutes when using hydrothermally synthesised Sn-Beta, while more than $60 \%$ methyl fructosides can be produced within few minutes using post synthetically synthesised Sn-Beta. A significant fraction of substrate thus is masked by rapid methyl fructoside formation prior to subsequent slow release of fructose. This release is the rate limiting step in the Sn-Beta catalysed methyl lactate process, but can be accelerated by the addition of small amounts of water at the expense of maximum methyl lactate yield.
\end{abstract}

\section{Introduction}

Simple sugars can be converted into fuels and chemicals, including levulinic acid, ${ }^{1-6} 5$-hydroxymethyl furfural (HMF), ${ }^{5,9}$ lactic acid $^{10,11}$ and others ${ }^{9,12,13,14,15-19}$ using zeolite-based materials as heterogeneous catalysts. Sn-Beta in particular is able to promote the cleavage, isomerization and dehydration of carbohydrates into more valuable chemicals. Such reactions include valorisations of abundant simple sugars by converting them to rare sugars ${ }^{9,11,17-25}$ at moderate temperatures near $100{ }^{\circ} \mathrm{C}$, or the formation of different hydroxyl esters as prospective building block for the production of biomassderived polymers at temperatures near $160^{\circ} \mathrm{C} .{ }^{13,14,16,26}$ Since the stability of the catalysts for the production of Sn-Beta is increased in alcoholic solvents as compared to water, ${ }^{6}$ the high temperature process is normally carried out in short-chain alcohol. The use of alcoholic solvents leads to the formation of activated and pH-neutral methyl esters and glycoside

\footnotetext{
a. Department of Chemistry, Technical University of Denmark, Kemitorvet, $2800 \mathrm{Kgs}$. Lyngby, Denmark, E-mail: ar@kemi.dtu.dk, semei@kemi.dtu.dk

${ }^{b}$ Haldor Topsøe A/S, Haldor Topsøes Alle 1, 2800 Kgs. Lyngby, Denmark.

c. Department of Electrical Engineering, Technical University of Denmark, $2800 \mathrm{Kgs}$. Lyngby, Denmark

+ Electronic Supplementary Information (ESI) available. See DOI: $10.1039 / \mathrm{x} 0 \times x \times 0000 x$
}

byproducts. ${ }^{12,17,19}$ The transformation of simple sugars into methyl lactate ${ }^{10,11}$ or other $\alpha$-hydroxy esters ${ }^{12-14,16,26}$ is a possible route for the sustainable production of polymers in bio-based processes. PLA (polylactic acid) has attracted great interest because of its mechanical and physical properties, the possibility to combine lactic acid with other monomers and to thus obtain a large variety of copolymer materials for diverse applications. ${ }^{27}$ In addition, lactic acid is a platform for other chemicals and alky lactates are promising green solvents. ${ }^{17}$

Knowledge-based approaches to improving biomass conversion processes could benefit from detailed kinetic and mechanistic understanding of the processes. Relatively little attention has been devoted to the systematic study of reaction kinetics in the Sn-Beta catalysed methyl lactate process. Herein, we therefore employ quantitative NMR methodology to derive a kinetic model of carbohydrate influx into the pathway that ultimately converts fructose intermediate to methyl lactate. Glucose and fructose had previously been shown to yield similar product mixtures in the Sn-Beta catalysed methyl lactate process, while the non-reducing disaccharide sucrose resulted in higher methyl lactate yield, possibly due to a slower release of reducing sugar. We hypothesised that a slow release of reducing sugar may play a generally neglected role in Sn-Beta catalysed conversions of glucose, fructose and sucrose at temperatures near $160{ }^{\circ} \mathrm{C}$, masking all of these substrates as methyl fructoside. Previous 


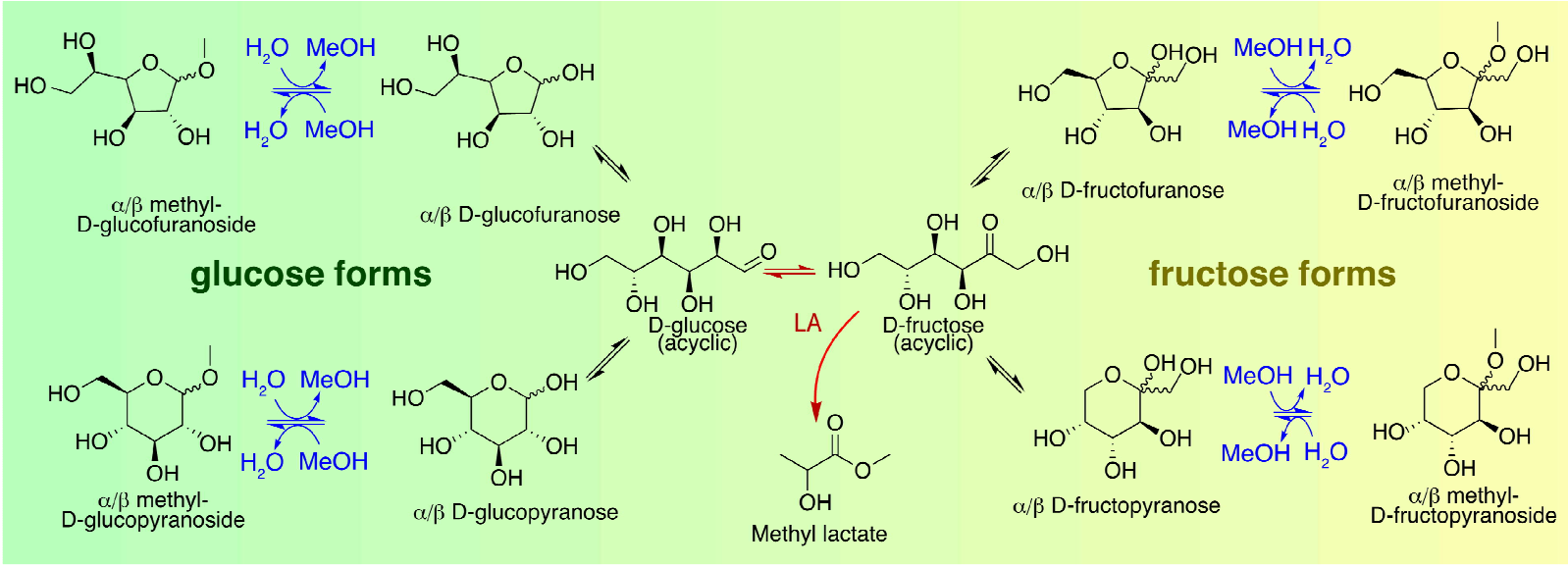

Scheme 1 Equilibria between glucose and fructose species in methanol. Lewis acidic active sites catalyse the isomerization of glucose and fructose as well as retroaldol cleavage to form methyl lactate (red arrows). Brønsted acidic sites promote the formation and hydrolysis of methyl glycosides (blue arrows). All these
sugars are present in the mixtures in their five and six-membered ring and $\alpha$-and $\beta$-forms.

results at $100{ }^{\circ} \mathrm{C}$ had shown that $\mathrm{Sn}$-Beta and other catalysts with Lewis and weak Brønsted acidity sequester carbohydrate as methyl-fructosides on the hours time scale. ${ }^{19}$ These processes would be predicted to operate on the minutes time scale at $160{ }^{\circ} \mathrm{C}$ in competition to retroaldol cleavage of fructose intermediate, and thus could impact on reaction kinetics and substrate influx into the methyl lactate process.

Scheme 1 depicts the equilibrium reactions of glucose and fructose in methanol in the presence of solid Lewis and Brønsted acid catalysts. Glucose and fructose are interconverted via isomerization through 1,2 hydride shift in the presence of Lewis acidic sites (red equilibrium arrows in Scheme 1), while Brønsted acidity promotes the formation of methyl glycosides. Fructose is generally considered the more reactive substrate than glucose due to its higher fraction of five membered and acyclic forms. ${ }^{22}$ In addition, the ketose to aldose isomerization of fructose or C1-C2 epimerization of glucose can also produce mannose. C6 sugars are mostly present as five- or six-membered rings termed furanose or pyranose forms, respectively. In the cyclic form, carbohydrates further exhibit stereoisomerism at the so-called anomeric position deriving from the carbonyl group with two possible anomeric forms called the $\alpha$ and $\beta$-forms. In the presence of Brønsted acidity in alcoholic solvents, five- or six-membered $\alpha$ and $\beta$-alkyl-glycosides are thus formed. Overall, a complex mixture of isomeric carbohydrates and glycosides is expected to form even from pure carbohydrate substrates in reactions using carbohydrate substrate, alcohol solvent and Sn-Beta catalyst above $100^{\circ} \mathrm{C}$ (Scheme 1 ).

We set out to study the formation of glycosides and how they affect the influx of carbon into the retroaldol-reactions. To this end, a quantitative high-resolution ${ }^{1} \mathrm{H}^{-13} \mathrm{C} 2 \mathrm{D} N \mathrm{NMR}$ approach was conducted at high magnetic field strength. A kinetic model of the methyl lactate process is obtained. Mechanistic and kinetic insights are applied to show that exclusion of water can warrant a process allowing high conversion of glucose and sucrose to methyl fructoside within as little as five minutes at $160^{\circ} \mathrm{C}$, especially when using Sn-Beta that contains defect sites. In the presence of added water at levels that comply with catalyst stability, substrate masking and reaction kinetics can be modulated to achieve faster formation of methyl lactate.

\section{Experimental Procedures}

\section{Catalysts preparation}

Sn-Beta zeolite was prepared via hydrothermal route as previously described. ${ }^{28,29}$ A mixture of $33.1 \mathrm{~g}$ of TEAOH (SigmaAldrich, $35 \%$ in water) and $30.6 \mathrm{~g}$ of TEOS (Sigma-Aldrich, $98 \%$ ) was magnetically stirred for $1 \mathrm{~h}$ until the appearance of a homogeneous phase. Then, a solution of $\mathrm{SnCl}_{4}{ }^{5} \mathrm{H}_{2} \mathrm{O}$ (SigmaAldrich, 98\%) in water was added with the proportion $\mathrm{Si} / \mathrm{Sn}=$ 150 and the mixture was kept stirring for $24 \mathrm{~h}$. Afterwards, 3.1 $\mathrm{g}$ of $\mathrm{HF}$ (Fluka, 47-51\%) in $1.6 \mathrm{~g}$ of $\mathrm{H}_{2} \mathrm{O}$ were added and homogenised under manual stirring. The white granulose solid was crystallised in a Teflon-lined stainless steel autoclave for 14 days at $140{ }^{\circ} \mathrm{C}$, filtered, washed thoroughly with $4 \mathrm{~L}$ of deionised water, dried at $80{ }^{\circ} \mathrm{C}$ overnight and finally calcined at $550{ }^{\circ} \mathrm{C}$ for $6 \mathrm{~h}$ to obtain HT Sn-Beta (150).

The Sn-Beta used for the synthesis of methyl fructosides was prepared via post-treatment (PT). ${ }^{30}$ Initially, $1 \mathrm{~g} \mathrm{H}$-Beta zeolite (Zeolyst, $\mathrm{Si} / \mathrm{Al}=12.5$, verified experimentally) was dealuminated by acidic treatment with $\mathrm{HNO}_{3}$ (VWR, 65\%, diluted to $13 \mathrm{M}$ in water) at $100{ }^{\circ} \mathrm{C}$ overnight. The deAl-Beta zeolite was then washed with water until neutral $\mathrm{pH}$ and dried overnight at $120^{\circ} \mathrm{C}$. Subsequently, the $\mathrm{Sn}$ was incorporated via incipient wetness impregnation using $\mathrm{SnCl}_{4} \cdot 5 \mathrm{H}_{2} \mathrm{O}$ dissolved in water added in a proportion for obtaining a Si/Sn ratio of 150 . The PT Sn-Beta (150) catalyst was finally dried overnight at 120 ${ }^{\circ} \mathrm{C}$ and calcined at $550{ }^{\circ} \mathrm{C}$ for $6 \mathrm{~h}$ before use. 


\section{Catalysts characterization}

Catalyst characterization included XRD, nitrogen physisorption and ICP-OES. Characterization by powder X-ray diffraction (XRD) was performed on an X'Pert diffractometer (Philips). XRD confirmed that the synthesis resulted in a Beta morphology without the formation of extra framework $\mathrm{SnO}_{2}$ (ESI, Fig. S1). Measurements of BET surface area and pore volume was performed by nitrogen adsorption/desorption measurements at $-196{ }^{\circ} \mathrm{C}$ using an Autosorb automatic surface area and pore size analyser (Quantachrome Instruments). The samples were heated at $200{ }^{\circ} \mathrm{C}$ for $4 \mathrm{~h}$ under vacuum before the nitrogen physisorption measurements. The elemental composition of the synthesised Sn-Beta materials was determined by inductively coupled plasma-atomic emission spectroscopy (ICP-OES) on a Perkin-Elmer Optima 3000 was used to determine the elemental composition of the hydrothermally and post-synthetically prepared Sn-Beta materials. The results of catalyst characterization are summarised in the ESI, Table S1. ${ }^{+}$Scanning Electron Microscope images were recorded on a FEI Quanta 200 ESEM FEG instrument (Fig. S3).

\section{Catalytic experiments}

The catalytic experiments were carried out in a Biotage ${ }^{\circledR}$ Initiator+ microwave synthesizer. D-(-)-fructose (Sigma-Aldrich, 99.9\%), D-(+)-glucose (Sigma-Aldrich, 99.5\%) and sucrose (Sigma-Aldrich, 99.5\%) were used as substrates. First, the sugar was solubilised in methanol (Sigma-Aldrich, 99.9\%) to yield stock solutions of $0.132 \mathrm{M}$ monomer concentration (0.066 M sucrose). Subsequently, $5 \mathrm{~mL}$ of stock solution and 50 $\mathrm{mg}$ of catalyst were reacted at $160^{\circ} \mathrm{C}$ under magnetic stirring for different times using $80 \mu \mathrm{L}$ of DMSO (Sigma-Aldrich, 99.5\%) as internal standard. In a typical time-resolved experiment, 10 reactions using the same conditions were carried out with reaction times of $5 \mathrm{~s}, 10 \mathrm{~s}, 30 \mathrm{~s}, 90 \mathrm{~s}, 5 \mathrm{~min}, 10 \mathrm{~min}, 20 \mathrm{~min}, 40$ $\mathrm{min}, 60 \mathrm{~min}$ and $240 \mathrm{~min}$. After the reaction, the catalyst was removed by filtration using a $0.22 \mu \mathrm{m}$ Nylon syringe filter (Frisenette) and the reaction mixture analysed using NMR spectroscopy.

\section{NMR spectroscopy}

NMR spectra were recorded on a Bruker Avance III 800 $\mathrm{MHz}$ spectrometer equipped with a $\mathrm{TCl}$ cryoprobe at $25{ }^{\circ} \mathrm{C}$. Crude reaction mixtures in protonated anhydrous methanol (Sigma-Aldrich, 99.8\%) were analysed after addition of $100 \mu \mathrm{L}$ of deuterated methanol (Sigma-Aldrich, $99.8 \% \mathrm{D}$ ) to $500 \mu \mathrm{L}$ of the reaction mixture. The spectra that were acquired included a quantitative $1 \mathrm{D}{ }^{13} \mathrm{C}$ spectrum. This spectrum was acquired with a recycle delay of $30 \mathrm{~s}$, sampling 65536 complex data points during an acquisition time of $1.36 \mathrm{~s}$. Quantitative ${ }^{13} \mathrm{C}$ NMR spectroscopy was pursued by the comparison of ${ }^{13} \mathrm{C} N M R$ signal areas in calibration samples relative to an internal standard (protonated DMSO). ${ }^{14}$

In order to improve sensitivity and reduce time requirements, more sensitive ${ }^{1} \mathrm{H}^{13} \mathrm{C}$ HSQC spectra were subsequently acquired on the calibration samples in order to obtain response factors for the quantitative analysis of the analytes of interest by the 2D HSQC spectra. ${ }^{1} \mathrm{H}-{ }^{13} \mathrm{C}-\mathrm{HSQC}$ spectra were acquired including a spectrum sampling $1024\left({ }^{1} \mathrm{H}\right) \times 300\left({ }^{13} \mathrm{C}\right)$ complex data points centered around a ${ }^{13} \mathrm{C}$ carrier offset of $62 \mathrm{ppm}$ and employing a spectral width of 20 $\mathrm{ppm}$ in the ${ }^{13} \mathrm{C}$ dimension to sample ${ }^{13} \mathrm{C}$ signal for $50 \mathrm{~ms}$. This spectrum of 25 min duration provided sufficient resolution and sensitivity to identify and quantify sucrose as well as glucose and fructose anomeric and ring forms as well as the anomeric and ring forms of their epimers and glycosides. To this end, signals of the $\mathrm{C} 6$ position were used as structural reporters. For the quantification of the sugars, the yield was calculated after measuring their response factor from signal areas in the ${ }^{1} \mathrm{H}-{ }^{13} \mathrm{C}$ HSQC spectral region of the primary alcohol (see Fig. 1B). Reaction mixtures were analysed in this way without further purification or derivatization, owing to the high resolution of ${ }^{1} \mathrm{H}^{13} \mathrm{C}$ HSQC spectra on mixtures of small molecule analytes when acquiring ${ }^{13} \mathrm{C}$ signal for sufficiently long acquisition times. ${ }^{31,32}$ Equivalently, ${ }^{1} \mathrm{H}-{ }^{13} \mathrm{C}-\mathrm{HSOC}$ spectra were acquired around a ${ }^{13} \mathrm{C}$ carrier offset of $102 \mathrm{ppm}$ in order to detect hemiacetal and acetal signals.

\section{Spectral processing and kinetic fitting}

All spectra were processed with ample zero filling in all dimensions and analysed in Bruker TopSpin 3.5pl7. Spectra were integrated and Methyl lactate integrals were fitted to kinetic rate laws in profit 6.29 (Quantumsoft), while glucose, fructose, fructoside and methyl lactate signals were fitted to a system of differential equations for the kinetic model of Fig. 5.

Kinetic rate laws for mono- and biexponential fits of methyl lactate yields $Y$ were as follows: For the monoexponential fit $\mathrm{Y}=\mathrm{A}\left(1-\mathrm{e}^{-\mathrm{kt}}\right)$, where $\mathrm{A}$ is the maximum yield and $k$ is the apparent first order rate constant. For the biexponential fit $\mathrm{Y}=\mathrm{B}\left(1-\mathrm{e}^{-\mathrm{tt}}\right)+\mathrm{C}\left(1-\mathrm{e}^{-\mathrm{mt}}\right)$, where $\mathrm{B}$ is the maximum yield of methyl lactate formed from fructose with apparent first order rate constant $I$ and $C$ is the maximum yield of methyl lactate formed from methyl fructoside with apparent first order rate constant $m$.

The system of differential equations for the kinetic model of Fig. 5 comprised the equations

(1) $d[G I c] / d t=-k_{1}[G I c]+k_{-1}[F r u]$

(2) $d[F r u] / d t=k_{1}[G I c]-k_{-1}[F r u]-k_{2}[F r u]+k_{-2}[\mathrm{Me}-F r u]-k_{3}[F r u]$

(3) $d[$ Me-Fru $] / d t=k_{2}\left[\right.$ Fru] $-k_{-2}[$ Me-Fru]

(4) $\mathrm{d}[\mathrm{Me}-\mathrm{Lac}] / \mathrm{dt}=\mathrm{k}_{3}[$ Fru]

where [Glc], [Fru], [Me-Fru] and [Me-Lac] are normalised integrals of glucose, fructose, methyl fructoside and methyl lactate, respectively. Byproducts such as methyl glucosides or reducing and glycoside forms of mannose and sorbose were omitted due to their low prevalence of few percent in the reaction mixture at any given time. Integrals were normalised to account for the loss of carbon into other byproducts and all integrals were weighted equally. Parameters $k_{1}, k_{-1}, k_{2}, k_{-2}$ and $k_{3}$ are rate constants that were determined by curve-fitting using Lmfit in the spyder (release 3.2.4) Python environment 
package. The kinetic parameters of equations (1)-(4) are defined in Fig. 5.

\section{Results and Discussion}

Chemical species detected in 2D NMR $\left({ }^{1} \mathrm{H}^{13} \mathrm{C} \mathrm{HSQC}\right)$ spectra of reaction mixtures of the $\mathrm{Sn}$-Beta catalysed conversion of hexoses to methyl lactate are exemplified in Fig. 1. Beyond glucosides and fructosides, mannoside as well as acetal forms of 3-deoxyglucosone signals were detected. In addition, sorbose was formed by 1,5 -hydride transfer from glucose g $^{33,34}$ and was usually present in the reaction mixture for the first few minutes in very small amounts $(<1 \%)$. Mannose, which is formed via a 1,2-carbon shift from glucose or by 1,2-hydride shift from fructose (Fig. S4), ${ }^{33,}{ }^{34}$ was most abundant at the beginning of the reaction with $<4 \%$ of maximum yield after few seconds of reaction from fructose, but was subsequently converted further, analogously to other sugars. Time resolved experiments using NMR spectroscopic detection akin to Fig. 1 were used to pursue a robust kinetic understanding of the methyl lactate process.
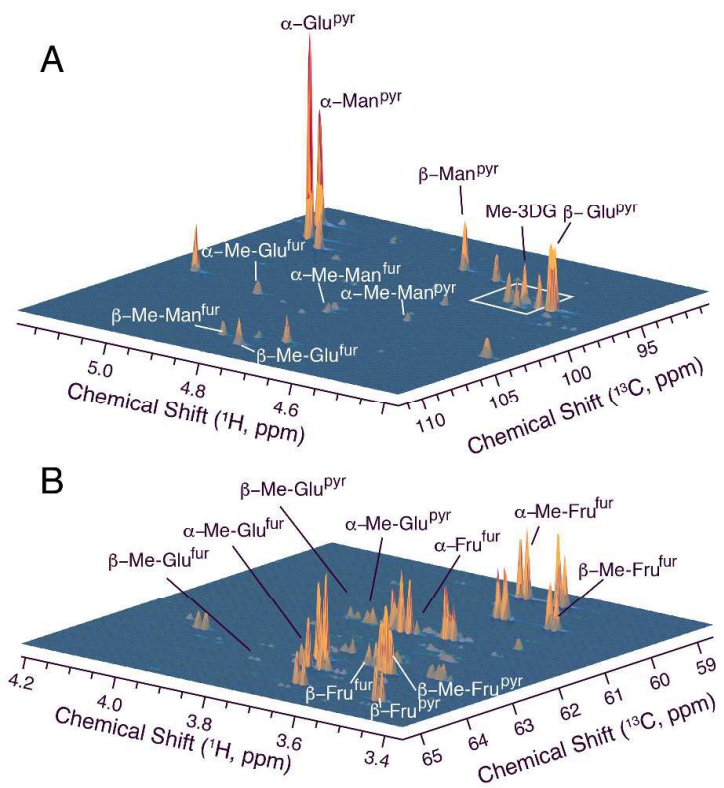

Fig. 1 Regions of ${ }^{1} \mathrm{H}-{ }^{13} \mathrm{C}-\mathrm{HSQC}$ spectra containing the anomeric $\mathrm{C} 1-\mathrm{H} 1$ groups of aldoses (A) and the primary alcohol $\mathrm{C} 6-\mathrm{H} 6$ groups of aldoses and ketoses (B) Detected signals are exemplified for the conversion of $120 \mathrm{mg}$ fructose in $5 \mathrm{~mL}$ methanol after $10 \mathrm{~s}$ at $160^{\circ} \mathrm{C}$ using a MW reactor. Abbreviations: Furanose (fur) pyranose (pyr), 3-deoxyglucosone (3DG), fructose (fru), glucose (glu), mannose (man).
Kinetic analysis of methyl lactate formation: Two regimes due to substrate masking as methyl fructoside

The chemocatalytic conversion of abundant carbohydrates to methyl lactate may benefit from a detailed understanding of rate limiting steps in the process in order to become more competitive to biocatalytic processes and provide optimised kinetics or yields. Analysis of reaction mixtures by quantitative ex situ NMR allowed the study of reaction progress. Fig. 2 displays the kinetics of methyl lactate formation from glucose alongside mono- and biexponential fits of the data. The data and kinetic fitting showed that methyl lactate was formed in a reaction encompassing two kinetic regimes. The initial fast regime accounted only for a minority of the methyl lactate formed and was followed by an orders of magnitude slower second regime. These data indicated that the majority of methyl lactate derived from a form of the substrate that was masked (protected in situ) in the chemocatalytic methyl lactate process catalysed by Sn-Beta in methanol.

The kinetic experiment of Fig. 2 was subsequently analysed for the conversion of glucose and the formation of other chemicals than methyl lactate over time. In addition, the kinetics of glucose, fructose and sucrose conversion were

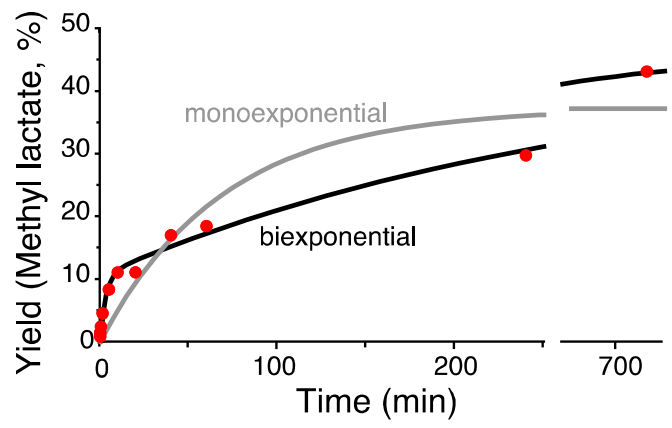

Fig. 2 Yield of methyl lactate from glucose in time resolved experiments. Fits of mono- and biexponential kinetics to the data are displayed, showing that methyl lactate forms in two kinetic regimes. Conditions: $120 \mathrm{mg}$ glucose, $50 \mathrm{mg} \mathrm{HT} \mathrm{Sn-}$
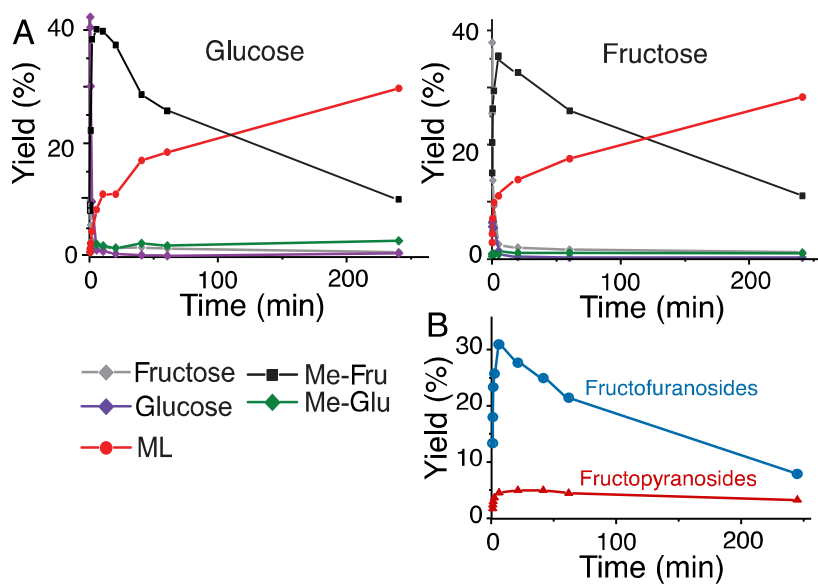

Fig. 3 Yields of glucose, fructose, methyl glycosides and methyl lactate in time resolved experiments starting from glucose and fructose. Conditions: $120 \mathrm{mg}$ cated time at $160^{\circ} \mathrm{C}$ 
compared, as shown in Fig. 3 and Fig. S5. Glucose and fructose reached comparable methyl lactate levels and comparable levels of methyl fructosides within $5 \mathrm{~min}$. On the order of $40-50 \%$ of all substrate was sequestered as methyl fructosides in the Sn-Beta catalysed conversion of glucose, fructose (Fig. 3A) and sucrose (Fig. S5), when using HT Sn-Beta at $160{ }^{\circ} \mathrm{C}$. After $5 \mathrm{~min}$, a slow decline of methyl fructosides was observed that correlated with the formation of methyl lactate in the slower reaction regime. The carbohydrate composition resulting from fructose and glucose substrate is compared in Fig. 3. As Sn-Beta is highly active in the isomerization of glucose to fructose, only a minor dependence on the starting substrate was observed.

Molecular detail on the fructoside formation was further probed by the distinction of the formed fructosides. Shape selectivity of zeolite catalysts could favor the formation of particular glycoside ring sizes. The kinetic experiment of Fig. 3B showed, however, that five-membered rings (furanosides) are the main forms as expected for Brønsted acid catalysed glycosidation under kinetic reaction control. During the time course of the reaction, the relative fraction of fructopyranosides increased, as expected due to the equilibration of the faster forming furanosides with pyranoside forms ${ }^{35}$ and due to faster hydrolysis of fructofuranosides. ${ }^{36}$ Notwithstanding, fructofuranosides remained the main form of masked substrate throughout the first 4 hours of the reaction.

\section{Different reactivity of glucose, fructose and sucrose}

The kinetics of conversion for glucose, fructose and sucrose substrate was subsequently compared (Fig. 4A). Albeit the SnBeta-catalysed conversion of carbohydrates is often conducted for several hours, the substrates are consumed on the seconds to minutes time scale. Fructose was converted more rapidly than glucose, while sucrose got converted slowest. Thus, sucrose solvolysis and glucose to fructose isomerization preceded subsequent reactions of fructose. Fructose is the most reactive species both in Brønsted acid catalysed formation of glycoside and in Lewis acid catalysed retroaldol cleavage. It is worth noting that higher yields of methyl lactate from sucrose than from glucose and fructose had previously been observed and had been ascribed to sucrose releasing both sugars more slowly from the disaccharide. ${ }^{37}$ Beyond the slower conversion of sucrose, we detected higher methyl fructoside levels from sucrose than from glucose and fructose substrate (Fig. S5). Such increased formation of methyl fructoside from sucrose indeed implies a particularly high degree of substrate masking using sucrose substrate and a low availability of free fructose.

Initial processes in the conversion of the monosaccharides glucose and fructose were deduced from kinetic profiles for the first $10 \mathrm{~min}$ of the reaction (Fig. 4B). Due to the higher availability of free fructose, methyl lactate and methyl
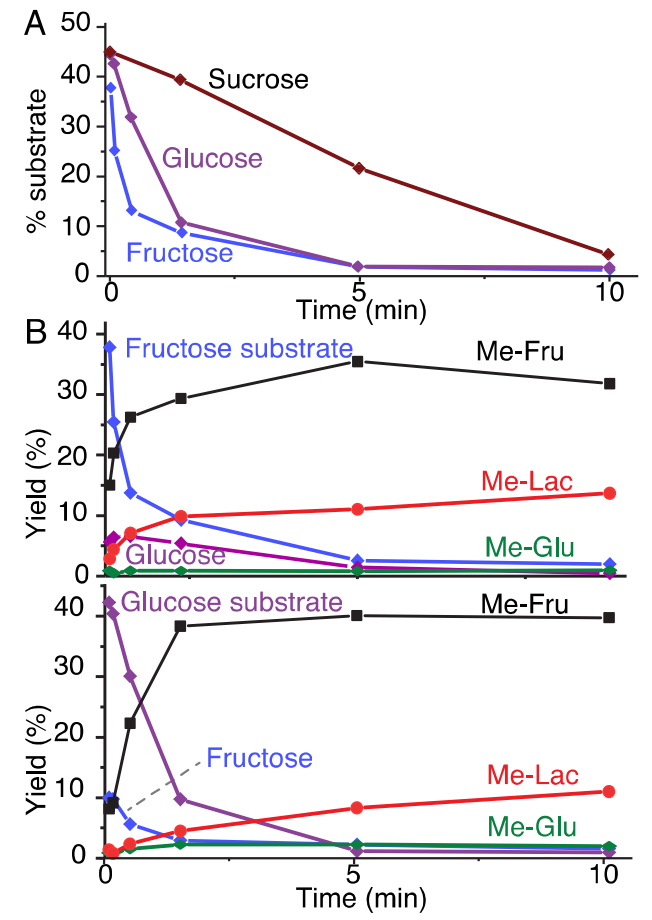

Fig. 4 (A) Comparison of fructose, glucose, and sucrose conversion. (B) Fructose and glucose conversion and yields of early intermediates in the time-resolved (150) catalyst, $5 \mathrm{~mL}$ methanol, $\mathrm{MW}$ reactor for the indicated time at $160^{\circ} \mathrm{C}$.

fructoside formed faster from fructose than from glucose in the initial rapid regime of methyl lactate formation (Fig. 4B). Methyl glucosides were formed in the reaction mixtures both from glucose and from fructose in yields lower than $3 \%$ by residual Brønsted acidity. The high Lewis acidity of Sn-Beta catalysed the isomerization between glucose and fructose, but led to an initial accumulation of the isomeric tautomer only to a yield of $7-10 \%$ due to the rapid masking of fructose as fructosides (Fig. 4B). Glucose-to-fructose isomerization thus avoids the accumulation of the thermodynamically stable glucosides $^{20}$ due to the kinetically preferred sequestration of fructosides both from glucose and fructose substrate. ${ }^{19}$

\section{Kinetic model}

Time-resolved experimental data permit proposing a plausible kinetic model for the methyl lactate process: Glucose and fructose isomerize rapidly. Fructose can undergo Lewis acidcatalysed retro-aldol cleavage and form methyl lactate or it can undergo Brønsted acid-catalysed masking with methanol to form methyl fructoside and water. This latter reaction is reversible under the reaction conditions, while the conversion to methyl lactate is irreversible.

A corresponding model with five kinetic rate constants is shown in Fig. 5A. The time-resolved experimental data for glucose conversion by HT Sn-Beta at $160{ }^{\circ} \mathrm{C}$ were fit to the kinetic model of glucose conversion to yield fits displayed in Fig. 5B and Fig. S6. The kinetic model fitted the data reasonably well. Glucose and fructose isomerised with rate constants $\mathrm{k}_{1}$ and $\mathrm{k}_{-1}$ of approximately $1.8 \mathrm{~min}^{-1}$. Fructose can 

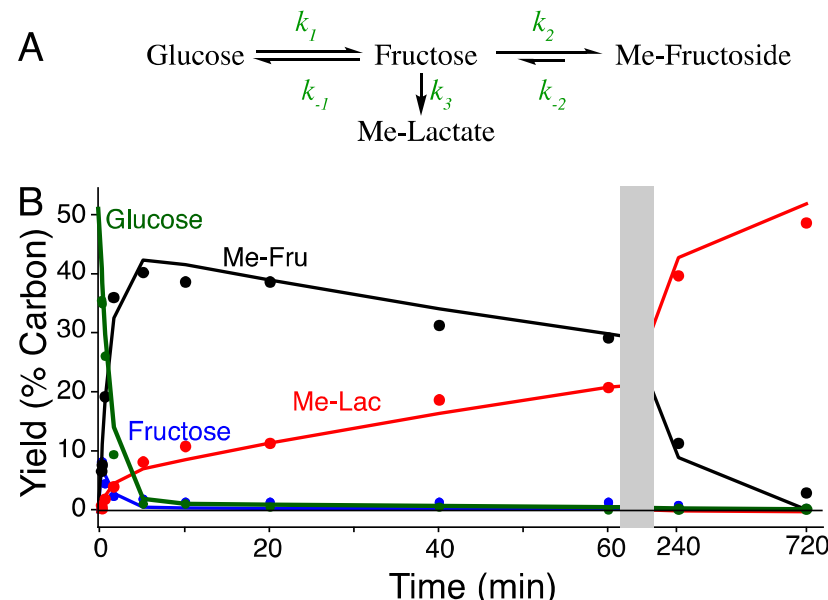

Fig. 5 (A) Reaction model for the conversion of glucose to methyl fructoside and methyl lactate. (B) Fit of experimental glucose, fructose, methyl fructoside and methyl lactate concentrations in time resolved experiments to the model of (A) the

also react to form methyl fructoside and methyl lactate. These products form in parallel as competing products of Lewis acid- and Brønsted acid-catalysed reactions. Formation of methyl fructoside was faster $\left(k_{2}=3.6 \mathrm{~min}^{-1}\right)$ than of methyl lactate $\left(k_{2}=0.72 \mathrm{~min}^{-1}\right)$, consistent with a higher conversion of fructose to fructoside than to methyl lactate in the initial fast regime of the methyl lactate process. Hydrolysis of methyl fructoside was limiting, with a rate $\mathrm{k}_{-2}$ of approximately 0.036 $\mathrm{min}^{-1}$, a factor of 20 slower than fructose conversion to methyl lactate. Hence, the methyl lactate process proceeds in two different kinetic regimes, as the fructose intermediate can react in a Lewis acid catalysed pathway to methyl lactate, but gets predominantly masked as methyl fructoside due to faster Fischer glycosidation by weak Brønsted acidity. Masked substrate subsequently reacted more than one order of magnitude slower due to rate limiting unmasking by methyl fructoside hydrolysis. The reaction scheme and rates thus indicated that addition of water could be used to influence unmasking and hence affect pathway kinetics in the methyl lactate process. In addition, the high yield of methyl fructoside within few minutes and its slower conversion indicated that a glucose to fructoside conversion process is feasible at $160{ }^{\circ} \mathrm{C}$ using catalysts with suitably balanced Brønsted and Lewis acidity.

\section{Glucose isomerization to $60 \%$ fructoside within few minutes}

The faster formation of methyl fructoside than of methyl lactate opens a possibility for production of methyl fructosides from glucose on the low minute timescale (rate constant of 3.6 $\min ^{-1}$ at $160{ }^{\circ} \mathrm{C}$ using HT Sn-Beta). Improved conversion of glucose to fructoside should be especially feasible when tailoring Brønsted and Lewis acidic sites to sequester fructose more efficiently as fructosides. To this end, we used a post

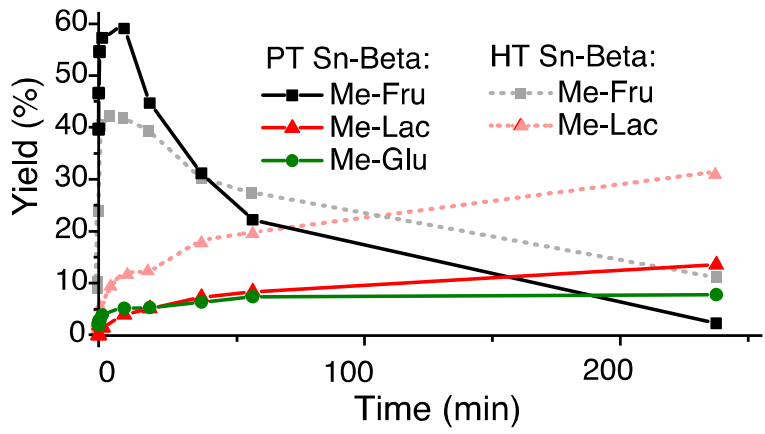

Fig. 6 Yields of methyl glycosides and methyl lactate from glucose in time resolved experiments using post treated Sn-Beta catalyst. Conditions: $120 \mathrm{mg}$
substrate, $50 \mathrm{mg}$ PT Sn-Beta (150) catalyst, $5 \mathrm{~mL}$ methanol, MW reactor for the substrate, $50 \mathrm{mg}$ PT Sn-Beta (150) catalyst, $5 \mathrm{~mL}$ methanol, MW reactor for the overlayed for comparison.

treated Sn-Beta catalyst. Higher Brønsted acidity in this defectcontaining PT Sn-Beta catalyst led to a higher accumulation of methyl glucosides than for the HT Sn-Beta. Notably, sufficient Lewis acidity to warrant rapid isomerization and higher Brønsted acidity resulted in higher levels of methyl fructosides when using the PT Sn-Beta catalyst, than when using the HT Sn-Beta catalyst (Fig. 6). Yields of $60 \%$ methyl fructoside could thus be achieved within 5-10 min from glucose at $160{ }^{\circ} \mathrm{C}$ using PT Sn-Beta, while the competing formation of methyl lactate was reduced relative to Brønsted acid catalysed Fischer glycosidation. These data showed that balanced Lewis and Brønsted acidity permit glucose to fructose isomerization at competitive yields within few minutes at $160{ }^{\circ} \mathrm{C}$, especially when using PT Sn-Beta.

While fructosides are reactive forms of masked substrate, glucosides accumulate on the low hour time scale and glucoside formation results in a deactivation of the substrate in the methyl lactate process (Fig. 6). These findings were validated by the use of commercial methyl glucoside as substrate for the methyl lactate process, showing that water contents above $75 \%$ were required to unmask significant fractions of methyl glucosides on the hours timescale. Such a high water content compromises catalyst stability at $160{ }^{\circ} \mathrm{C}$ and is thus not desirable.

\section{Effect of water on rate and yield of methyl lactate formation}

Kinetic data of the methyl lactate process showed that substrate masking occurred, and that unmasking of methyl fructoside by hydrolysis was rate limiting for the formation of the majority of methyl lactate. Hence, we probed the effect of water addition on kinetics and yields of the methyl lactate process, using glucose as the substrate and water levels that complied with catalyst stability at $160{ }^{\circ} \mathrm{C}$. We hypothesised that small amounts of water would aid the unmasking of methyl fructoside and thus accelerate the formation of methyl lactate. Fig. 7A shows the kinetic profile of methyl fructoside in the presence of varying amounts of water. The masking of substrate in the form of fructosides decreased in the presence 

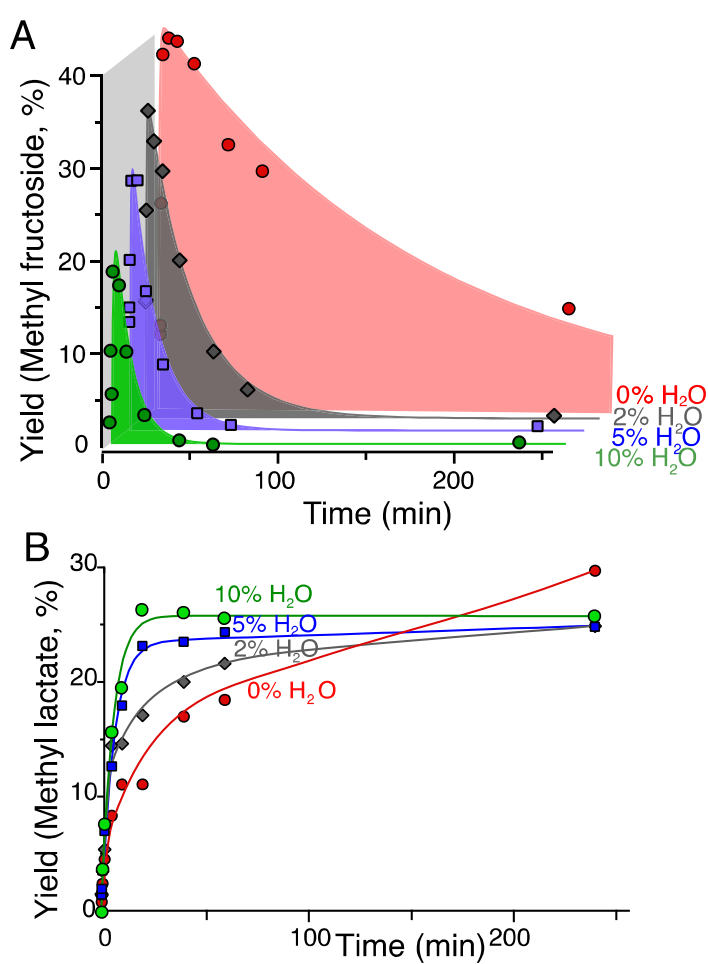

Fig. 7 (A) Methyl fructoside formation and consumption using glucose as the substrate in the presence of different amounts of water in methanol $5 \mathrm{~mL}$ total volume) at $160^{\circ} \mathrm{C}$ and $50 \mathrm{mg}$ HT Sn-Beta catalyst. Accumulation of methy fructosides decreases as the water content increases. (B) Faster formation of methyl lactate in the presence of water, and higher final yield in the absence of
water. The sum yield of lactate and methyl lactate is displayed.

of water as anticipated. This decrease in the accumulation of methyl fructoside correlated with faster hydrolysis of methyl fructoside. Thus, the addition of $2 \%, 5 \%$ and $10 \%$ water accelerated the hydrolysis of methyl fructosides by factors of 5.6, 9.9 and 15.5, respectively. In this manner, the addition of water at moderate concentrations that are not obstructive to catalyst stability could accelerate the mobilization of the masked methyl fructoside in the chemocatalytic conversion of carbohydrates in methanol.

Rates of methyl lactate formation were increased in the presence of increasing amounts of water, even though water may be expected to compete with the binding of substrate to the catalyst $\mathrm{Sn}$ sites. $^{38}$ Notably, the reduced masking of substrate in the presence of added water was accompanied by lower final yields of methyl lactate (Fig. 7B). In addition, reduced methyl fructoside pools in the presence of added water largely reduced the slow kinetic regime and increased the fraction of methyl lactate formed in the initial fast regime. As a result, it took approximately 70, 35, 12 and 7 minutes to reach a yield of $20 \%$ methyl lactate upon addition of $0 \%, 2 \%$, $5 \%$ and $10 \%$ water, respectively (Fig. 7B). Higher water contents were not studied, as they are detrimental to catalyst stability $^{39}$ and to final methyl lactate yields (Fig. 7B), but favor formation of carbonaceous material and of Brønsted acid catalyzed products. ${ }^{10}$ Furthermore, methyl fructoside was
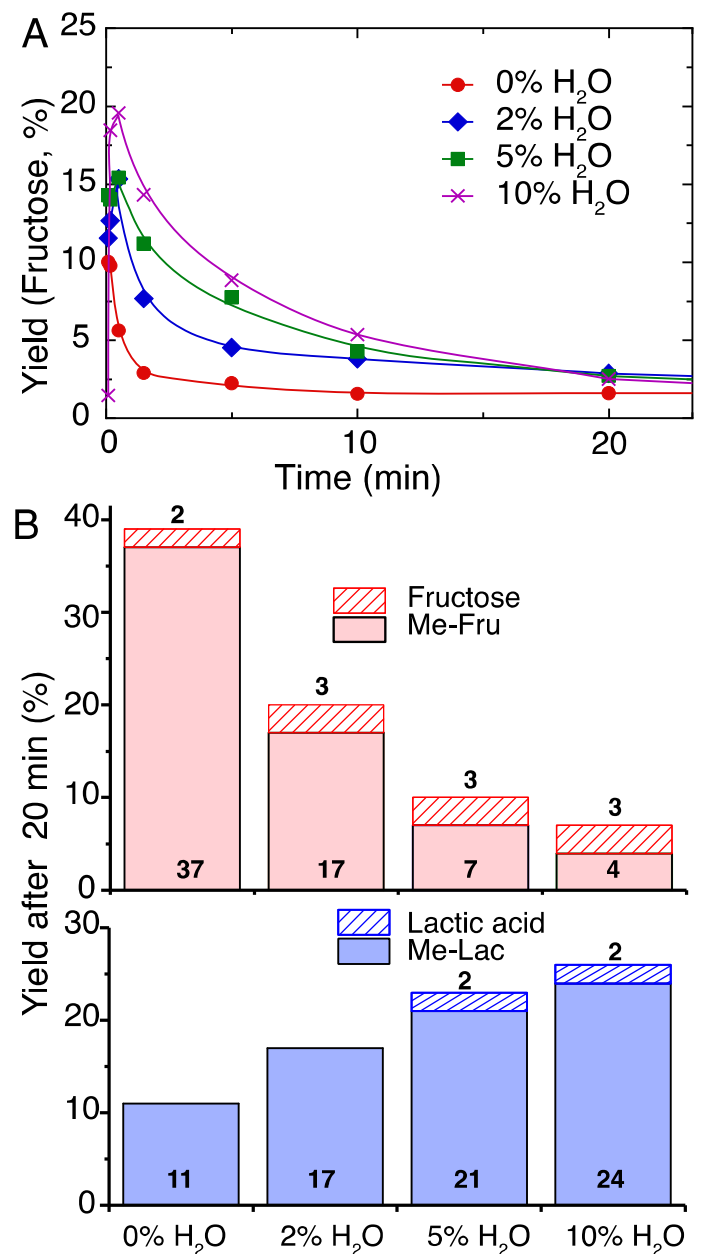

Fig. 8. (A) Fructose formation from glucose in the presence of different amounts of water. (B) Methyl fructoside and methyl lactate formation from glucose within 20 minutes of reaction time in the presence of varying amounts of water. .

quantitatively mobilized within 40 minutes at water concentrations as low as 5\% (Fig. 7A). These results are consistent with recent findings indicating that the methyl lactate process under flow conditions is best performed in the presence of water in the $1-10 \%$ range, with an optimum near $5 \% .^{39}$

Increased unmasking of methyl fructoside and faster formation of methyl lactate correlated to increased free fructose intermediate in the presence of added water (Fig. 8A). The increased availability of free fructose during the first 20 min of reaction allowed methyl lactate yields to be reached in the presence of $5-10 \%(\mathrm{v} / \mathrm{v})$ water that would take hours to achieve in the absence of added water (Fig. 7B). These findings indicate that the yield of methyl lactate per unit time could be optimised by water addition, with possible implications for the production capacity both by batch and flow setups. Yields of methyl lactate, lactate, methyl fructoside and fructose after 20 min are summarised in Fig. 8B. Notably, only minor amounts of lactate were formed relative to the predominant methyl lactate product, in the range of $0-10 \%$ added water. Similar results were obtained when using fructose instead of glucose 
as the substrate. Also in this case, fructoside formation was suppressed by the addition of small amounts of water, while the conversion to methyl lactate was accelerated in the presence of water (Fig. S7). These reactions of fructose underline possible benefits of water addition in improving the production of methyl lactate per unit time by modulating kinetic profiles of the methyl lactate process with water.

\section{Conclusions}

This work describes kinetic and mechanistic insight into the by Sn-Beta catalysed methyl lactate process in methanol with an emphasis of carbohydrate influx into the pathway. Plausible kinetic differences in the conversion of glucose, fructose and sucrose can be detected, while methyl lactate formation from all substrates shows two different kinetic regimes. The majority of methyl lactate is formed in the slow reaction regime. This slow regime results from the accumulation of methyl fructoside (especially in its furanoside form) as a masked form of substrate carbon for glucose, fructose and sucrose. Sucrose, known to yield higher methyl lactate levels than glucose and fructose, also yields higher methyl fructoside levels. Kinetic insight is practically applied by showing that the fast methyl fructoside formation and its slow hydrolysis can be used to produce more than $60 \%$ fructoside within few minutes using a defect-containing Sn-Beta catalyst. In addition, masking of substrate as methyl fructoside permits modulating process kinetics by the presence of water. The conversion to methyl lactate can thus be accelerated by the addition of small amounts of water that are not prohibitive to catalyst stability. Such small amounts of water may in some instances be endemic to the process, if carbohydrate substrate is supplied as syrup. The findings described herein have implications for strategies to improve and tailor the methyl lactate process, specifically in increasing productivity in the presence of water and for the rapid isomerization of glucose to methyl fructosides at high temperature in the absence of water.

\section{Conflicts of interest}

There are no conflicts to declare.

\section{Acknowledgements}

We gratefully acknowledge funding by the Innovation Fund Denmark (case number 5150-00023B). $800 \mathrm{MHz}$ NMR spectra were recorded on the spectrometer of the DTU NMR center.

1 J. Jow, G. L. Rorrer, M. C. Hawley and D. T. A. Lamport, Biomass, 1987, 14, 185-194.

2 H. Li, Z. Fang, J. Luo and S. Yang, Appl. Catal. B: Environ., 2017, 200, 182-191.

3 L. Peng, H. Li, L. Lin and K. Chen, Catal. Commun., 2015, 59, 10-13.
4 B. Girisuta, L. P. B. M. Janssen and H. J. Heeres, Chem. Eng. Res. Des., 2006, 84, 339-349.

5 V. Choudhary, S. H. Mushrif, C. Ho, A. Anderko, V. Nikolakis, N. S. Marinkovic, A. I. Frenkel, S. I. Sandler and D. G. Vlachos, J. Am. Chem. Soc., 2013, 135, 3997-4006.

6 S. Saravanamurugan and A. Riisager, ChemCatChem, 2013, 5, 1754-1757.

7 T. Wang, M. W. Nolte and B. H. Shanks, Green Chem., 2014, 16, 548-572.

8 R.-J. van Putten, J. C. van der Waal, E. de Jong, C. B. Rasrendra, H. J. Heeres and J. G. de Vries, Chemical Rev., 2013, 113, 1499-1597.

9 M. Moliner, Y. Román-Leshkov and M. E. Davis, Proc. Nat. Acad. Sci., 2010, 107, 6164-6168.

10 M. S. Holm, S. Saravanamurugan and E. Taarning, Science, 2010, 328, 602-605.

11 E. Taarning, S. Saravanamurugan, M. S. Holm, J. Xiong, R. M. West and C. H. Christensen, ChemSusChem, 2009, 2, 625627.

12 S. Tolborg, S. Meier, I. Sadaba, S. G. Elliot, S. K. Kristensen, S. Saravanamurugan, A. Riisager, P. Fristrup, T. Skrydstrup and E. Taarning, Green Chem., 2016, 18, 3360-3369.

13 S. G. Elliot, C. Andersen, S. Tolborg, S. Meier, I. Sádaba, A. E. Daugaard and E. Taarning, RSC Adv., 2017, 7, 985-996.

14 S. G. Elliot, S. Tolborg, I. Sádaba, E. Taarning and S. Meier, ChemSusChem, 2017, 10, 2990-2996.

15 H. S. Chen, A. Wang, H. Sorek, J. D. Lewis, Y. Román-Leshkov and A. Bell, ChemistrySelect, 2016, 1, 4167-4172.

16 R. De Clercq, M. Dusselier and B. F. Sels, Green Chem., 2017, 19, 5012-5040.

17 M. Paniagua, S. Saravanamurugan, M. Melian-Rodriguez, J. A. Melero and A. Riisager, ChemSusChem, 2015, 8, 10881094.

18 S. Saravanamurugan, M. Paniagua, J. A. Melero and A. Riisager, J. Am. Chem. Soc., 2013, 135, 5246-5249.

19 S. Saravanamurugan, I. Tosi, K. H. Rasmussen, R. E. Jensen, E. Taarning, S. Meier and A. Riisager, Catal. Sci. Techn., 2017, 7, 2782-2788.

20 S. Saravanamurugan, A. Riisager, E. Taarning and S. Meier, Chem CatChem, 2016, 8, 3107-3111.

21 Y. Román-Leshkov, M. Moliner, J. A. Labinger and M. E. Davis, Angew. Chem. Int. Ed., 2010, 49, 8954-8957.

22 R. Bermejo-Deval, R. S. Assary, E. Nikolla, M. Moliner, Y. Román-Leshkov, S.-J. Hwang, A. Palsdottir, D. Silverman, R. F. Lobo, L. A. Curtiss and M. E. Davis, Proc. Nat. Acad. Sci., 2012, 109, 9727-9732.

23 J. Dijkmans, D. Gabriels, M. Dusselier, F. de Clippel, P. Vanelderen, K. Houthoofd, A. Malfliet, Y. Pontikes and B. F. Sels, Green Chem., 2013, 15, 2777-2785.

24 N. Rajabbeigi, A. I. Torres, C. M. Lew, B. Elyassi, L. Ren, Z. Wang, H. Je Cho, W. Fan, P. Daoutidis and M. Tsapatsis, Chem. Eng. Sci., 2014, 116, 235-242.

25 S. Li, T. Josephson, D. G. Vlachos and S. Caratzoulas, J. Catal., 2017, 355, 11-16.

26 M. Dusselier, P. Van Wouwe, S. De Smet, R. De Clercq, L. Verbelen, P. Van Puyvelde, F. E. Du Prez and B. F. Sels, ACS Catal., 2013, 3, 1786-1800.

27 S. Farah, D. G. Anderson and R. Langer, Adv. Drug Delivery Rev., 2016, 107, 367-392.

28 S. Tolborg, A. Katerinopoulou, D. D. Falcone, I. Sadaba, C. M. Osmundsen, R. J. Davis, E. Taarning, P. Fristrup and M. S. Holm, J. Mat. Chem. A, 2014, 2, 20252-20262.

29 A. Corma, L. T. Nemeth, M. Renz and S. Valencia, Nature, $2001,412,423$.

30 C. Hammond, S. Conrad and I. Hermans, Angew. Chem. Int. Ed., 2012, 51, 11736-11739.

31 M. Bøjstrup, B. O. Petersen, S. R. Beeren, O. Hindsgaul and S. Meier, Anal. Chem., 2013, 85, 8802-8808. 
32 B. O. Petersen, O. Hindsgaul and S. Meier, Analyst, 2014, 139, 401-406.

33 H. Nguyen, V. Nikolakis and D. G. Vlachos, ACS Catal., 2016, 6, 1497-1504.

34 R. Bermejo-Deval, R. Gounder and M. E. Davis, ACS Catal., 2012, 2, 2705-2713.

35 G. S. Bethell and R. J. Ferrier, Carbohydrate Res., 1973, 31, 69-80.

36 L. J. Heidt and C. B. Purves, J. Am. Chem. Soc., 1938, 60, 1206-1210.
37 M. Dusselier and B. F. Sels, in Selective Catalysis for Renewable Feedstocks and Chemicals, ed. K. M. Nicholas, Springer International Publishing, Cham, 2014, DOI: 10.1007/128_2014_540, pp. 85-125.

38 W. N. P. van der Graaff, C. H. L. Tempelman, G. Li, B. Mezari, N. Kosinov, E. A. Pidko and E. J. M. Hensen, ChemSusChem, 2016, 9, 3145-3149.

39 D. Padovan, S. Tolborg, L. Botti, E. Taarning, I. Sadaba and C. Hammond, Reaction Chemistry \& Engineering, 2018, DOI: 10.1039/C7RE00180K. 


\section{TOC entry}

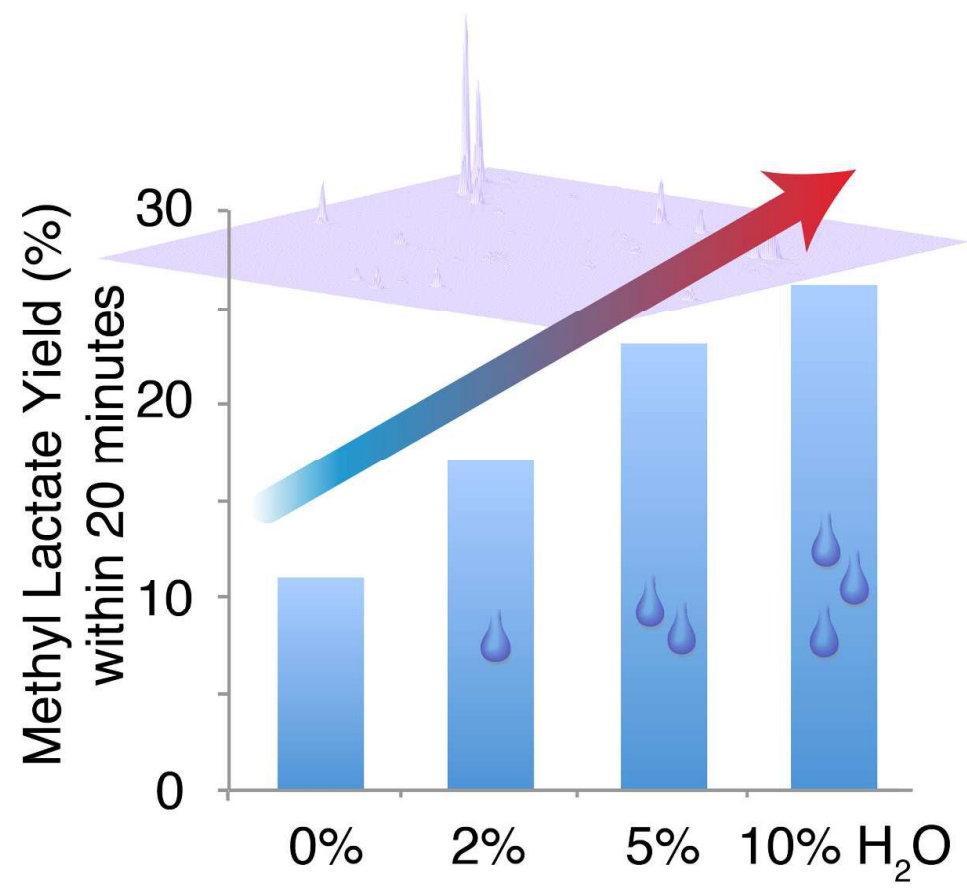

Strategies to tailor the Sn-Beta catalysed methyl lactate process are identified by kinetic and mechanistic insight. 